\title{
La demanda como aspecto metodológico de la extensión universitaria a partir de un avance de investigación en Carreras de Ingeniería
}

\section{The demand as an aspect of methodology of universitary extension from the start point of an advancement in research at the Faculty of Engineering}

\author{
Marcelo Luís López \\ Docente de la Facultad de Humanidades \\ Universidad Nacional de Jujuy \\ San Salvador de Jujuy, Argentina \\ marllopez@yahoo.es
}

Recibido: 07-IX-2010 • Aceptado 26-X-2010 • Corregido 23-VI-2011

\begin{abstract}
Resumen: El trabajo corresponde a un avance de investigación que, desde un enfoque cualitativo, aborda el problema del tratamiento que tiene la demanda en materia de Extensión Universitaria en distintos ámbitos de la Universidad Pública Estatal de Jujuy, en el noroeste argentino. Ello se realiza con el objetivo de analizar y vincular estilos de abordaje de la demanda con procesos y resultados utilizando como procedimiento de base para la recolección de información entrevistas a agentes extensionistas y miembros de organizaciones impactadas. Se presentan resultados preliminares de las primeras entrevistas realizadas en el ámbito de las ingenierías, para lo cual se examinaron 8 precategorias semisetructurantes de las entrevistas: 1) concepto de extensión que orienta la práctica; 2) ámbitos, organizaciones y agentes con los que se realiza la vinculación; 3) características asignadas a los demandantes; 4) criterios para clasificar pedidos y acciones; 5) formas y vías como se tratan estos pedidos; 6) formación sobre demanda; 7) estrategias extensionistas; 8) actividades de alto/bajo impacto evocadas en materia de extensión. Además, se encontraron códigos emergentes que junto con las anteriores permitieron establecer cuatro categorías relevantes para el análisis de la demanda en extensión
\end{abstract}

\section{Introducción}

El trabajo corresponde a un avance de la investigación "La Demanda en Extensión Universitaria en la Universidad Nacional de Jujuy"1, que tiene como unidad de origen a uno de los equipos de investigación de la cátedra de Residencia de Ciencias de la Educación de la Facultad de Humanidades y Ciencias Sociales (FHYCS) de la Universidad Nacional de Jujuy (UNJU).

La Universidad Nacional de Jujuy se ubica en la provincia de Jujuy al noroeste de la República Argentina. Fue creada en 1973 funcionando su rectorado en el edificio de la "Misión Universitaria de Estudios de Patología Regional", donde desarrolló sus trabajos sobre el mal de Chagas el célebre Salvador Mazza ${ }^{2}$. 
(académica, política, normativa y económica), con derivaciones metodológicas para la actividad extensionista.

Palabras clave: Extensión Universitaria, Problema de la demanda, Carreras de ingeniería

\begin{abstract}
The work is an advance of an investigation. From a qualitative perspective analyzes the problem of treating the demand on University Extension in different areas of the University of Jujuy (argentinean northwest). The objectives are to relate styles demand approach to processes and results. Shown here is preliminary analysis for the area of engineering. Were interviewed extension agents. We examined eight categories of the interviews: 1) The concept of extension, 2) Organizations with which the connection is made, 3) features assigned to the applicants, 4) Criteria for classifying the actions, 5) processing of orders, 6) Extension training on demand, 7) extension strategies used, 8) activities of high or low impact in terms of extension. Emerging codes were also found, which together allowed us to establish four new categories relevant to the analysis of demand in extent, with their respective properties (academic, political, regulatory and economic) with methodological derivations for Extension.
\end{abstract}

Keywords: Extension, Social demand, Racing Engineering
Posee otras tres facultades además de la FHYCS (Ingeniería, Ciencias Agrarias y Ciencias Económicas) y una "Escuela de Minas" de nivel medio (preparatoria) en una provincia que tiene un perfil históricoproductivo con identidad en la minería, la explotación azucarera y la producción tabacalera. En conjunto, todas estas unidades atienden una matrícula de 12.966 alumnos ${ }^{3}$ en el marco de una población general para la provincia de 611.484 habitantes (según datos del el último censo de 2001).

El problema de investigación abordado es: ¿qué tratamiento tiene la demanda en materia de Extensión en distintos ámbitos de la Universidad Pública Estatal de Jujuy y cuál es el rol de la teoría al respecto? El objetivo de la investigación es intentar una primera aproximación a la identificación y análisis de estilos de abordaje de la demanda y sus posibles vínculos con procesos y resultados en materia de Extensión con las especificidades propias de las facultades que componen la UNJU, así como aportar a la problemática metodológica de la Extensión Universitaria.

Se parte de un posicionamiento de la Extensión como interacción Social (López, 2005, p.15; Quiroga, 2001; p.1) en que se considera a la función de Extensión Universitaria $\mathrm{u}$ otros equivalentes conceptuales como tercera función de la educación Superior (las otras dos son docencia e investigación $\square$ López, 2010, p. $2 \square$ ), que involucra acciones con sentido político para el desarrollo social, cultural y productivo y la superación de la injusticia y la desigualdad en una construcción compartida entre la universidad y el medio intra y extra universitario, funciones sobre las que además el discurso universitario viene construyendo su identidad para diferenciarse de otros niveles educativos.

El concepto de demanda fue desarrollado principalmente desde enfoques teóricos de la Psicología Institucional y el Análisis Institucional considerando que la misma tiene como emergentes pedidos o encargos que hacen individuos $\mathrm{u}$ organizaciones, $\mathrm{y} / \mathrm{o}$ 
las definiciones que ciertos grupos realizan con base en diagnósticos para intervenir sobre otros grupos. Tales enunciaciones/ acciones se articulan sobre deseos y necesidades con contenidos manifiestos y objetivos explícitos que constituyen los emergentes de la demanda -su parte visible-, detrás de los cuales puede haber una trama más compleja de contenidos latentes, objetivos implícitos y otros actores disimulados u omitidos, aspectos que pueden ser parcialmente develados para romper con la ingenuidad y la tentación de efectuar intervenciones que finalmente puedan tener poca relevancia. Por otra parte, se relaciona con lo que un grupo debería acceder, lo que efectivamente pide y la transición de lo privado o sectorial a la esfera de lo público y el bien común; es decir, que se convierta en una cuestión social (Bleger, 1999, p. 58; Butelman, 1987, p. 28; Fernández, 2003, p. 231; Lourau, 1991, p. 190; Pampliega de Quiroga, 1994, p. 37; Pichon Riviere, 1985, p. 17; Sirvent, 1996$, p. 77$)^{4}$. Esta tarea de ayudar a la explicitación de la demanda y de dar respuesta a los pedidos en su mayor complejidad, integrándolos a la agenda política, es entendida aquí como un papel fundamental de la tarea extensionista desde una perspectiva de interacción social.

Se espera que al examinar la problemática de la demanda en extensión, aun tratándose de un recorte centrado en carreras de Ingeniería, se pueda contribuir con una referencia orientadora para la didáctica de los aspectos extensionistas de las cátedras que realizan simultáneamente tareas de docencia y extensión, es decir devoluciones al medio durante la formación, como por ejemplo en salidas al campo o en las Residencias Docentes o Profesionales (instancia de origen de este trabajo). En este tipo de espacios curriculares frecuentemente se da respuesta a solicitudes de organizaciones del medio, al tiempo que se realizan prácticas, pero muchas veces de manera lineal, sin connotar las mismas o sin considerarlas como posibles síntomas de problemas mayores, lo que determina que las intervenciones no produzcan los impactos esperados ni satisfagan las expectativas de las partes involucradas.

Por ello puede ser superador contar con esbozos de teorías provisorias que surjan de la propia práctica vinculándolas con otras teorías como la de la demanda que, en este y otros trabajos, se viene pretendiendo articular con la Extensión Universitaria. Aunque en el discurso universitario la Extensión se encuentra equiparada a las otras funciones de la universidad, posee muchas menos ofertas y aportes para la formación tanto a nivel curricular como extracurricular, por lo que los intentos de ampliación de su base metodológica (como este) constituyen un adicional potencial para la pedagogía de la Extensión.

\section{Aspectos metodológicos}

La perspectiva epistemológica del trabajo es interpretativa. El interés es comprender la práctica extensionista situada, profundizando el abordaje e interpretación de relatos de hechos y situaciones más que obteniendo explicaciones a partir de la generalización de una gran cantidad de sucesos. Ello se hace desde un diseño flexible (Mendizabal, 2007, p. 67) con un correlato metodológico cualitativo en que se trata de construir una trama textual que relacione la narración de actividades desarrolladas por los actores involucrados en las etapas iniciales o de refundación de procesos extensionistas, con el sentido asignado por ellos a su accionar. Esto se efectúa dentro de un diseño no experimental basado en la teoría fundamentada y lo que se ha dado en denominar como el paradigma de codificación que prosigue una secuencia retroalimentable de codificación abierta, axial y selectiva con la elaboración de categorías, propiedades y dimensiones durante la reducción analítica del material (Soneira, 2007, p. 153; Rodríguez Gómez, Gil Flores y García Jiménez, 1996, p. 48). 
Para tal fin se utilizaron como procedimiento de base para la recolección de información entrevistas a una muestra intencional significativa de agentes extensionistas y miembros de organizaciones impactadas. En el presente trabajo se anticipan resultados preliminares de las primeras incursiones a carreras de Ingeniería por medio de cuatro entrevistas realizadas a informantes clave ${ }^{5}$ dentro de las prácticas extensionistas de la Facultad de Ingeniería (FI) y otras cuatro en la Facultad de Ciencias Agrarias (FCA), ocho en total, con relación a los siguientes ocho ejes que semiestructuraron las entrevistas (además del reagrupamiento de etiquetas y códigos en categorías emergentes): 1) concepto de extensión que orienta la práctica; 2) ámbitos, organizaciones y agentes con los que se realiza la vinculación; 3) características asignadas a los demandantes; 4) criterios para clasificar pedidos y acciones; 5) formas y vías como se tratan pedidos y acciones; 6) formación sobre demanda; 7) estrategias extensionistas; 8) actividades de alto/bajo impacto evocadas en materia de extensión.

La perspectiva epistemológica del trabajo es interpretativa. El interés es comprender la práctica extensionista situada, profundizando el abordaje e interpretación de relatos de hechos y situaciones más que obteniendo explicaciones a partir de la generalización de una gran cantidad de sucesos. Ello se hace desde un diseño flexible (Mendizabal, 2007, p. 67) con un correlato metodológico cualitativo en que se trata de construir una trama textual que relacione la narración de actividades desarrolladas por los actores involucrados en las etapas iniciales o de refundación de procesos extensionistas, con el sentido asignado por ellos a su accionar. Esto se efectúa dentro de un diseño no experimental basado en la teoría fundamentada y lo que se ha dado en denominar como el paradigma de codificación que prosigue una secuencia retroalimentable de codificación abierta, axial y selectiva con la elaboración de categorías, propiedades y dimensiones durante la reducción analítica del material (Soneira, 2007, p. 153; Rodríguez Gómez, Gil Flores y García Jiménez, 1996, p. 48).

Para tal fin se utilizaron como procedimiento de base para la recolección de información entrevistas a una muestra intencional significativa de agentes extensionistas y miembros de organizaciones impactadas. En el presente trabajo se anticipan resultados preliminares de las primeras incursiones a carreras de Ingeniería por medio de cuatro entrevistas realizadas a informantes clave dentro de las prácticas extensionistas de la Facultad de Ingeniería (FI) y otras cuatro en la Facultad de Ciencias Agrarias (FCA), ocho en total, con relación a los siguientes ocho ejes que semiestructuraron las entrevistas (además del reagrupamiento de etiquetas y códigos en categorías emergentes): 1) concepto de extensión que orienta la práctica; 2) ámbitos, organizaciones y agentes con los que se realiza la vinculación; 3) características asignadas a los demandantes; 4) criterios para clasificar pedidos y acciones; 5) formas y vías como se tratan pedidos y acciones; 6) formación sobre demanda; 7) estrategias extensionistas; 8) actividades de alto/bajo impacto evocadas en materia de extensión.

\section{Interpretación de precategorías $y$ categorías emergentes}

En la línea de lo que plantea M. T. Sirvent (1997, p. 9), las categorías están referidas a "las partes de un concepto que orientan el juicio científico" y que "delimitan su dominio, concretan el enfoque con que se usa el concepto". El concepto central que se pretende dilucidar a través de una investigación es el objeto de investigación, que para este trabajo es la demanda en Extensión, lo que a su vez permite construir justificaciones respecto de la pertinencia de 
las categorías: si las mismas están presentes o no, su calidad, jerarquía y la forma y/o grado de manifestación para ésta o futuras investigaciones. Las categorías a priori o precategorías son las que se estiman como relevantes de antemano mientras que las emergentes son las que surgen de un análisis expo facto por la importancia que argumenta el investigador y/o la redundancia y saturación de las etiquetas y códigos que en principio constituyen los primeros elementos provisorios, que surgen de las apreciaciones durante el análisis, en el intento de reducir la información recolectada a un núcleo de material significativo con relación al problema que se investiga (a lo largo de un mismo registro o de varios y distintos registros de entrevistas, observaciones, etc.).

\subsection{Precategoría: concepto de extensión que orienta la práctica}

Se etiquetaron 29 alusiones, fragmentos de entrevista, que permitieron el siguiente análisis (se presentan primero los fragmentos de material discursivo por una cuestión metodológica respecto que de allí se extraen los nuevos códigos y categorías que se ubican inmediatamente a continuación): ${ }^{6}$

"la función de extensión es transferir los conocimientos" (Ent. 1 p. 1).

"la extensión es estee digamos llevarle a alguien un conocimiento." (Ent. 4, p. 1).

"En ese sentido a la Extensión yo la veo como casi... una transferencia al medio." "De nuestros trabajos de nuestros conocimientos de lo que estamos haciendo en el campo de la ingeniería” (Ent. 6, p. 1).

En una codificación abierta se obtuvo como subcategoría de primer orden a la extensión como transferencia de conocimientos al medio que incluye como subcategorías de segundo orden a:

"interpretaba que la Extensión Universitaria era un poco la difusión de, de actividades que no estaban eh, involucradas por otras instituciones y la Universidad, digamos en cumplimiento de un rol eh... cultural" "ofrecer actividades culturales, eh, artísticas, que un poco era la actividad de la Casa de la Cultura de la Universidad Nacional de Jujuy, hoy la actividad de la Casa de la Cultura con sus talleres de teatro, talleres de pintura" (Ent. 5 p. 1).

"nosotros tenemos... digamos, llamémosle, al concepto... no vamos específicamente a lo cultural como es mayormente en gran parte de la universidad argentina que le hace, le adjudica a la extensión únicamente una función eminentemente cultural.” (Ent. 8, p. 1).

3.1.1 La difusión cultural que se valora o se desestima, según sea el caso, pero que en definitiva se reconoce aunque no específicamente ligada al ámbito de las ingenierías;

"nosotros como grupo de investigación trabajamos con campesinado con productores aborígenes donde la problemática no es tan sencilla como la de la falta de una tecnología o sea que el productor no se ha enterado o de cierta tecnología" (Ent. 4, p. 1).

"los primeros seis meses del año 2003 hubo a nivel Nacional una... digamos una movilización muy importante en cuanto a la legitimación de la universidad frente a la sociedad...” “...el Dr. Duhalde cuando, cuando asumió la presidencia y destacó o puso en valor digamos el rol de la Universidad como consultor del Estado...” “...el aspecto de vinculación tecnológica era una zona gris que... que las universidades nacionales no habían, digamos, tomado en general...", “.. muy... limitados a ciertas universidades” (Ent. 5, p. 2). "hay que formar una unidad paralela de buena onda tipo fundación, cooperadora, una cosa así para encauzar por ese lado porque la universidad y alguna razón hay porquee... ante algún problema ante todo de transferencia en tecnología que pudiese llegar a dar algún error que incidiría en la vida de la gente" (Ent. 7, p. 1).

"Qué transferencia y qué podemos brindarle al medio en ese aspecto. Eso es un poco a la vinculación." "no solamente en las aulas o en las currículas" "todas las técnicas y todas las tecnologías que podamos transmitir”. (Ent. 6, p. 1).

\subsubsection{La vinculación tecnológica}

o vinculación con el medio cuando se incorporan a la escena contextos sociales, políticos, legales, productivos, etc. que articulan lo intra con la extra universitario.

"más allá de esta diferencia, este... conceptual que se a hecho y que se ha, digamos, interiorizado en las universidades, considero que la extensión incluye 
este aspecto, eh, hay muchos colegas, digamos, que hemos participado en estas redes de vinculación que marcaban la diferencia y que consideraban, que consideran, digamos, que no es la vinculación tecnológica un área de la extensión universitaria" "la vinculación tecnológica es una actividad que hace la extensión con, eh... la..., perdón, la universidad con el medio" (Ent. 5, p. 3).
Por otra parte, ciertos componentes de la relación extensión-vinculación mantienen una tensión dialéctica. A continuación se referencian algunas propiedades que caracterizan a la precategoría de partida y la subcategoría empírica emergente mencionada extensión como vinculación tecnológica y con el medio:

\begin{tabular}{lll}
\hline $\mathrm{N}^{\mathrm{o}}$ & \multicolumn{1}{c}{ Fragmentos de discurso } & \multicolumn{1}{c}{ Propiedad Abstraída } \\
\hline 1 & Transferencia a partir del proceso de investigación ligado a la & Clasificación de la extensión por su \\
& difusión a través de diferentes tipos de intervenciones (notas & origen y características: \\
& Ent. 3, sin referencia textual). & 1) innovaciones a partir de procesos \\
& "una de las características con Ingeniería es que la Extensión & de investigación; \\
& viene a través de pedidos de trabajos, servicio a terceros le & 2) servicios repetitivos a terceros; \\
& llamamos nosotros. (Ent. 6, p. 1). & 3) desarrollos adaptados a la \\
& "habría una línea que le llamaríamos servicios a terceros" "se & organización.
\end{tabular}
efectúan ensayos que muchas veces son digamos repetitivos, no son ensayos que requieran mucho desarrollo de técnicas operatorias para desarrollar los análisis" "Está el otro concepto que no está muy desarrollado pero que tiene que, ahí es donde está el meollo de la cuestión, es la vinculación con la investigación propiamente dicha o con el equipo investigador que ejecute su propio desarrollo ya sea porque lo requieran terceros, una empresa" (Ent. 8, p. 1).

"Demandas tenemos siempre por la predisposición pero no siempre podemos este digamos abordar estamos limitados a los ejes que trabajamos lógicamente pero demandas tenemos siempre y lo que podemos cooperamos con las comunidades en algunas cosas podemos avanzar mucho mas ahora estamos desarrollando dos pequeñas propuestas con dos comunidades que es un trabajo dual" (Ent. 4 p. 3).

"Claro la tercera modalidad para mí está" "Es un desarrollo que se hace en la empresa, con la empresa" (Ent. 8, p. 6).

"la extensión orgánica a través de la dependencia que corres-

2 ponde que es supuestamente tendría que ser la secretaria de extensión ya sea de la facultad o de la universidad es decir para que sea mejor" (Ent. 1 p. 1).

3 "Si nosotros una vez hicimos un bosquejo eeh, fundamentalmente de que la institución saliera digamos al medio digamos a ofrecer servicios no digamos servicios sino ofrecer su servicio para seleccionar la problemática por ahí muy puntual que tiene el productor" (Ent. 1 p. 7).

Clasificación de la extensión por el grado de institucionalización: en orgánica vs. inorgánica.

Objetivos / efectos de las acciones Brindar servicios y solucionar problemas 


\begin{tabular}{l}
\hline $\mathrm{N}^{\mathrm{o}}$ \\
\hline 4 \\
"Los niveles de lenguaje, hay que adecuarlo, todo" (Ent. 2, p. \\
10). \\
"Por otro lado nosotros consideramos la tecnología digamos \\
también no solo en el aspecto productivo sino en un marco \\
más amplio y la consideramos como un aspecto también de \\
expresión de la cultura digamos" (Ent. 4, p. 1). \\
"Estee ya del otro extremo, ¿entendés? O sea como fortalecer o \\
poner lo que la comunidad hace en el, bajo el enfoque nuestro \\
en el verdadero valor positivo que tiene y como la sociedad \\
mayor hace para que eso no desaparezca" "Cuando el enfoque \\
clásico era: estos son productores inservibles que hacen todo \\
mal y tienen que aprender todo" (Ent. 4 p. 7).
\end{tabular}

5 “extensión, creo que hoy la extensión universitaria digamos esta, eh... digamos, evolucionando en el sentido de que cada vez es más comprometido este, el papel de la universidad, la legitimación de la universidad” (Ent. 5, p. 3).

6 "La universidad debería pensar en armar una unidad este paralela de una fundación seria de manera que sustente ese tipo de actividad con total limitación de las responsabilidades jurídicas.” “...hoy en el día la Universidad de Jujuy en particular no tiene un sistema administrativo contable como para sostener un contrato de cierto nivel porque entre que paga sueldos y tiene las obligaciones, ahora con la obra no se como le estará yendo porque me parece que debe ser un laboreo bastante intenso para muy poca gente que hay, que esta abocada al sistema administrativo universitario específico, ¿no?” (Ent. 7, p. 2).

"Pongamos que alguna vez vamos a tener que dirigir proyectos o gente o enseñar postgrado y formar gente en este nivel, hay que conocer un poco política de Estado sino se pueden cometer algunos errores que son groseros y ahí vienen problemas que hay que tratar de evitar porque la sociedad es altamente especulativa, seguramente va a vivir de los errores que cometamos y en ingeniería es donde más errores se pueden cometer ante todo por no conocer un poco.

La política de Estado es bastante fría establece que las universidades no están preparadas ni eh instituidas para hacer transferencia..." (Ent. 7, p. 1).

\author{
Propiedad Abstraída
}

Dialéctica entre un enfoque clásico de extensión de actores inservibles que hay que enseñarles todo vs. un enfoque innovador que considera la retroalimentación entre la cultura universitaria y la cultura de las organizaciones impactadas

Rol de la Extensión como herramienta de legitimadora de la universidad frente a la sociedad

Derivaciones en la extensión de la organización de recursos financieros, respaldo jurídico y aspectos administrativos contables para realizar esta función desde la universidad frente a la política de Estado una sociedad especulativa 
Con relación a la propiedad 1 , los aspectos que potencialmente se pueden poner en una dimensión en que esta se expresa son:

\begin{tabular}{|c|c|}
\hline Dimensión & Ejemplo de fragmentos de discurso que dan sustentación \\
\hline $\begin{array}{l}\text { Certificaciones } \\
\text { (Rubro servicios) }\end{array}$ & $\begin{array}{l}\text { "hay docentes por cátedras no es cierto que por ejemplo salen a hacer un curso de capa- } \\
\text { citación agropecuario, y lo siguen haciendo hoy no, a riesgo propio, y sin saber digamos } \\
\text { o saben o desconocen o no quieren digamos conseguir la autorización pertinente que } \\
\text { corresponde a las autoridades de la facultad" “...después vienen y piden que se les certi- } \\
\text { fique entonces nosotros no podemos certificar algo que no sabemos." (Ent. } 1 \text { p. 1) } \\
\text { "Y bueno, luego seguimos, y seguimos con pies desbocados, ya habíamos hecho digamos } \\
\text { una referencia como certificadores, entonces después certificamos para eh... otras enfer- } \\
\text { medades." (Ent. } 2 \text { p. 2). }\end{array}$ \\
\hline Publicaciones & $\begin{array}{l}\text { "estudiamos la enfermedad, sacamos también en una pequeña publicación" (Ent. } 2 \text { p. } \\
\text { 2)." } \\
\text { "ahora estamos por sacar bueno hemos sacado un par de publicaciones y la idea es hacer } \\
\text { un libro, un librito sobre biodiversidad y sistemas familiares de producción" (Ent. 4, p. } \\
\text { 6). }\end{array}$ \\
\hline $\begin{array}{l}\text { Asesoramientos } \\
\text { (Rubro servicios) }\end{array}$ & $\begin{array}{l}\text { los asesoramos [a los productores] cuando hicieron la presentación de un protocolo para } \\
\text { exportar". (Ent. } 2 \text { p. } 2 \text { ). } \\
\text { Transferencia a partir del proceso de investigación ligado a la difusión a través de ase- } \\
\text { soramientos (notas Ent. } 3 \text {, sin referencia textual). }\end{array}$ \\
\hline $\begin{array}{l}\text { Laboratorio } \\
\text { (Innovaciones a } \\
\text { partir de procesos } \\
\text { de investigación) }\end{array}$ & $\begin{array}{l}\text { "tenemos el laboratorio donde trabajamos con microscopios, con estufas, hacemos deter- } \\
\text { minaciones que van más allá del diagnóstico de campo del profesional. Entonces, nues- } \\
\text { tros colegas, nuestros compañeros de la facultad mismo que están en el campo vienen y } \\
\text { consultan, también nos consultan" (Ent. } 2 \text { p. } 3 \text { ). } \\
\text { "habría una línea que le llamaríamos servicios a terceros que es de alguna manera el } \\
\text { trabajo que se hace a través de laboratorios" (Ent. } 8, \text { p. } 1 \text { ). }\end{array}$ \\
\hline $\begin{array}{l}\text { Capacitación } \\
\text { (Rubro servicios }+ \\
\text { Innovaciones a } \\
\text { partir de procesos } \\
\text { de investigación) }\end{array}$ & $\begin{array}{l}\text { "Entonces nosotros capacitábamos a los productores" (Ent. } 2 \text { p. 2). } \\
\text { Transferencia a partir del proceso de investigación ligado a la difusión a través de capa- } \\
\text { citaciones (notas Ent. } 3 \text {, sin referencia textual). } \\
\text { "para hacer transferencia, es un problema básico complejo desde ya que la única autori- } \\
\text { zación que hay es para dar cursos" (Ent. 7, p. 1). } \\
\text { "lo que hacemos como actividades de extensión es cursos para personas que no son } \\
\text { alumnos de la Facultad y que son del profesorado de nivel terciario cursos de, las únicas } \\
\text { actividades de extensión que hacemos son con relación a la enseñanza pero que son sí } \\
\text { cursos" (Ent. 5, p. 10). } \\
\text { "transferencia de conocimientos a través de cursos de capacitación hacia el medio no } \\
\text { solamente en las aulas o en las currículas que nosotros tenemos sino hacia el medio } \\
\text { como entonces de hecho es lo que nosotros sabemos es transferirlo al medio. (Ent. } 6 \text {, } \\
\text { p. 1). }\end{array}$ \\
\hline $\begin{array}{l}\text { Pasantías } \\
\text { (Rubro servicios) }\end{array}$ & $\begin{array}{l}\text { "ofrecer a nuestros alumnos la posibilidad de realizar pasantía íbamos a casi todas las } \\
\text { empresas de la provincia y reparticiones públicas" (Ent. } 5 \text {, p. 5). } \\
\text { "Vamos desde el tema este de las pasantías, que en este momento me estoy acordando" } \\
\text { (Ent. } 6, \text { p. 1). }\end{array}$ \\
\hline
\end{tabular}




\subsection{Precategoría: ámbitos, organizaciones y agentes con los que se realiza la vinculación}

Se etiquetaron 38 alusiones que los entrevistados realizan con relación a los ámbitos y organizaciones que demandan extensión a la universidad. Las mismas se reagruparon en un cuadro en el que se considera a cada una de las Facultades -Ingeniería y Ciencias Agrarias- en relación a ámbitos inespecíficos o generales y organizaciones concretas. Ello permite obtener un primer panorama parcial de algunas instituciones con las que se vincula la UNJU en el campo de las ingenierias:

\begin{tabular}{|c|c|c|}
\hline \multicolumn{3}{|c|}{ Articulación con } \\
\hline $\mathrm{N}^{\mathrm{o}}$ & Sectores amplios & Organizaciones concretas \\
\hline FCA & $\begin{array}{l}\text { Agentes turísticos. } \\
\text { Agricultura familiar pequeños, productores de } \\
\text { la quebrada de Humahuaca. } \\
\text { Algunas áreas geográficas impactadas: } \\
\text { Palmasola. Yuto. Maimará, Huacalera, } \\
\text { Cercanías de Humahuaca (Cianzo, Ocumazo, } \\
\text { San Roque), Cangrejillos, Yavi. } \\
\text { Asociaciones de productores (citricultores). } \\
\text { Cooperativas de Productores. } \\
\text { Municipios. } \\
\text { Pequeños agricultores de la quebrada. } \\
\text { Sector productivo agropecuario. }\end{array}$ & $\begin{array}{l}\text { AFINOA - Asociación Fitosanitaria del Noroeste } \\
\text { Argentino } \square \text {. } \\
\text { Cooperativa Cauqueva de Maimará. } \\
\text { Cooperativa de tabacaleros. } \\
\text { INET - Instituto Nacional de Educación } \\
\text { Tecnológica } \square \text {. } \\
\text { INTA - Instituto Nacional de Tecnología } \\
\text { Agropecuaria } \square \text {. } \\
\text { SENASA - Servicio Nacional de Sanidad y Calidad } \\
\text { Agroalimentaria } \square \text {. }\end{array}$ \\
\hline FI & $\begin{array}{l}\text { Centros vecinales. } \\
\text { Empresas mineras. } \\
\text { Ministerio de Trabajo de la Nación. } \\
\text { Ministerios de la Provincia de Jujuy: Gobierno } \\
\text { Educación, Producción, Medioambiente, } \\
\text { Infraestructura. } \\
\text { Organizaciones sociales. }\end{array}$ & $\begin{array}{l}\text { EXPOJUJUY (ocasional y periódico). } \\
\text { Ingenio Ledesma. } \\
\text { Autosol-Autolux. } \\
\text { Ticon. } \\
\text { SAE - Sociedad Argentina de Emergencias. } \\
\text { Unión Industrial de Palpalá y Jujuy. } \\
\text { Asociación de municipios. } \\
\text { Proyecto "Iniciativa Jujuy" (Organismo mixto } \\
\text { Estatal+Privado). } \\
\text { Planta de cemento Mineti. } \\
\text { ISJ - Instituto de Seguros de Jujuy. } \\
\text { Hospital san Roque. } \\
\text { Mineras: Pirquitas, Aguilar. } \\
\text { Agua de los Andes. }\end{array}$ \\
\hline
\end{tabular}

\subsection{Precategoría: características asignadas a los demandantes}

Se etiquetaron 19 alusiones. Del discurso de los entrevistados en la FCA surge la reagrupación de los demandantes en agentes rurales conformados por campesinos y aborígenes, por un lado, con las propiedades asignadas de poseer un nivel de lenguaje distinto a los extensionistas, una organización de trabajo familiar con predominio de tracción a sangre y trabajo manual (campesinos) sin pertenencia a organizaciones (en el caso de los aborígenes) que se mueven en áreas geográficas que funcionan de manera conservadora y tradicional, y que, a su vez, generan una dialéctica en los extensionistas de la universidad entre 
productor desinformado que necesita una psicología especial vs. modos de expresión de la cultura; por otro lado, técnicos y profesionales. Aparece un segundo grupo advenedizo que son los agentes turísticos, como guías de turismo, en que el objeto de la extensión tiene que ver especialmente con el rubro alimentos y cuya propiedad tiene que ver con un destinatario más instruido.

En la Facultad de Ingeniería, se identifican actores amplios como: 1) una "sociedad nueva" con demandas e intereses propios actuales con buena predisposición en la transferencia pero con comportamientos prebendarios que generan como contrapartida desconfianza en las autoridades (universitarias) para la articulación y una potencial actitud conservadora; 2) un Estado con recursos, burocrático e ineficiente que hace mal las cosas; 3) un sector productivo que se moderniza, que valoriza la imagen, con exigencias crecientes a medida que va obteniendo resultados en la articulación con la universidad.

\subsection{Precategorías: criterios para clasificar pedidos y acciones / tratamiento que se le da a los pedidos que llegan y que se clasifican así y vías por la que estos llegan}

Se fusionaron estas dos precategorías de partida (con un etiquetado en conjunto de 33 alusiones) porque durante el análisis se estimó que no se justificaba su escisión y cuyas propiedades se tratan a continuación.

Los entrevistados referencian el seguimiento de vías informales (lo que en el análisis de la categoría 1 se definía como extensión inorgánica) que resuelven los mismos agentes quienes a su vez creen que deberían salir más al medio a traer problemáticas que saben que existen para articular la búsqueda de soluciones mediante programación vs. pruebas y ensayos en que algunas cátedras tienen canales de comunicación aceitados. Por otra parte, están las vías formales (extensión orgánica) en que los niveles más altos de gestión y conducción toman la decisión produciendo pérdida de rol a las áreas de extensión o se sigue una vía despersonalizada en que se reemplazan vínculos por protocolos o formatos que proveen convocatorias. Sin embargo, símultáneamente hay quienes reconocen la funcionalidad e importancia para la clasificación de los pedidos del área de extensión. Esta pérdida de rol de las áreas de extensión para algunos entrevistados tendría como consecuencia la conformación de vínculos débiles con sectores de la comunidad lo que a su vez se identifica con la falta de estructura de las mismas para, entre otras cosas, conformar vías más institucionalizadas que permitan clasificar mejor y dar curso a la demanda extensionista.

A su vez, en los relatos de los interpelados, las vías formales pueden comenzar a partir de contactos informales como una primera etapa o directamente de proyectos formalizados como programas que vienen de organismos oficiales como la Secretaría de Políticas Universitarias.

\subsection{Precategoría: formación sobre demanda}

Aunque esta es una de las categorías más relevantes para la investigación, en las entrevistas realizadas en las ingenierías se encontró poco eco, a pesar de que sí una mención significativa referida a autoformación en psicología laboral.

\subsection{Precategoría: estrategias extensionistas}

Esta categoría a priori aunque puede tener aparente desconexión con el objeto de investigación, es decir la relación demandaextensión, su importancia deviene en que en entrevistas informales e intercambios con otros extensionistas, por ejemplo en congresos, muchos relacionan el tratamiento 
que le dan a la demanda con relación a las posibilidades procedimentales que prevén para llevar a cabo las potenciales intervenciones. Con un total de 50 etiquetas alusivas resultantes de la segmentación de las entrevistas, se subcategorizaron tres tipos de miradas relevantes sobre las estrategias que se denominaron: obstáculos en la metodología extensionista; estrategias relativamente conocidas y/o esperables; estrategias con algún componente innovador.

\subsubsection{Obstáculos en la metodolo- gía extensionista}

Sobre este eje se encontraron "sensaciones" en los entrevistados de estar frente a carencias metodológicas, escasa formación teórica y pocas publicaciones orientadoras al respecto, aunque también se piensan soluciones como el desarrollo de un posgrado de formación de vinculadores con capacidades en investigación y extensión con expectativas de obtener roles como el de un gerente de ventas (FI) $\square$ lo que a su vez refuerza la precategoría I respecto al tipo de concepciones de extensión que circulan en las Ingenierías $\square$.

\subsubsection{Estrategias relativamente conocidas y/o esperables}

Se reagrupó un listado de procedimientos que aparece en los dichos de los entrevistados como los siguientes.

Reuniones con productores (FCA), repartir folletos con la oferta educativa, participación e integración en actos/actividades sociales inespecíficas por parte de las autoridades de la facultad como estrategia para iniciar procesos de vinculación, trabajo de laboratorio, consultas, confección de páginas web para el intercambio comunicacional y publicaciones para la transferencia.

Otras cuestiones relevantes evocadas son: la necesidad de equipamiento que determina intercambio de asistencia por compra de equipos como una alternativa de financiamiento de las actividades de extensión; la firma de convenios y actas/ cartas acuerdos como estrategias de las UU.NN para brindar un marco legal orientador de los derechos y obligaciones de las partes; la valorización de la extensión a nivel de las universidades por los conocimientos valiosos que la sociedad necesita no sólo disciplinarios sino también conocimientos generales en una coyuntura de la Universidad Argentina que es creíble; vincular investigación con extensión y transferencia; la necesidad de conformar grupos y trabajar sobre la secuencia formación de gente $\square$ cambio generacional $\square$ preparar para gerenciar el propio emprendimiento, contrato, convenio.

\subsubsection{Estrategias con algún com- ponente innovador}

Acá se encontró un listado de cosas interesantes vividas como originales por los propios entrevistados y que poseen menor circulación en el discurso de los universitarios extensionistas de la UNJU como: el desarrollo de estrategias de capacitación teórico-prácticas para la gente de campo (FCA) en tres etapas, 1) introducción teórica, 2) material audiovisual, 3) trabajo con material concreto; la necesidad de un trabajo más interdisciplinario ya que en la FCA existe la dificultad de trabajar con comunidades que son muy cerradas, los líderes con más experiencia ofrecen mayor resistencia y esta aparece especialmente cuando la Universidad va para ciertas tareas y no hace devolución; esta situación llevó a que en la FCA se creara un Centro de Investigación y Desarrollo (CEIDA) para trabajar con pequeños productores donde se vinculan diferentes cátedras (semiinstitucionalizado sin presupuesto), el cual funciona como una estrategia para además romper la estructura vertical de las misms, lo que conlleva a la organización de seminarios internos para el trabajo interdisciplinar (en el camino de 
lo que puede llegar a ser transdisciplinar); otra estrategia es solicitar que la organización colabore con algún insumo en la intervención para tener mayor certidumbre de la autenticidad de la demanda y abaratar costos para facilitar/posibilitar las intervenciones y obtener un efecto multiplicador; la creación de estructuras que mejoren los aspectos organizativos y el impacto del accionar extensionista para lo que hay distintas propuestas como fundaciones $^{7}$ para sortear el obstáculo excesivamente burocrático del manejo de fondos centralizados desde el Rectorado, una estructura administrativa organizativa tipo una pequeña secretaría con un gerente designado por el decano para abordar el tema del ingreso de fondos y su distribución y/o una oficina (o sede) de unidad de Vinculación en cada Facultad (que actualmente centraliza rectorado), con un Consejo de Vinculación; otras dos ideas coadyuvantes a generar una política de extensión son la creación de un Consejo de Extensión Económico Social, en el cual participen actores diversos de la comunidad -gobierno, organizaciones sociales, cámaras empresariales- y Un Consejo de Extensión en el sentido del Consejo de Ciencia y Técnica de la UNJU que tiene representación de delegados por Facultad; también ubicar agentes claves tomando referentes en distintas unidades académicas que hubieran tenido alguna experiencia con Extensión asignando importancia al protagonismo del responsable de la extensión en la primera etapa - de las transferencias donde las empresas ponen recursos económicos- para evitar desconfianzas y que los proyectos luego se frenen por problemas de dosificación de los fondos (en ingeniería es difícil trabajar sin recursos porque los gastos son grandes y acumulativos).

Asimismo se propone la presencia de una persona con buena (in)formación por parte de los grupos de investigación y extensión para hacer el "marketing" y la figura de un docente colaborador adscripto (actualmente existe aprobada en la FI con número de resolución) que desarrolle tareas de extensión para el cuatrimestre que no se realiza dictado de clases; trabajar sobre modelos de transferencia "out sourcing" (basado en el conocimiento) en que la empresa financia el desarrollo de una unidad en la universidad para temas específicos que esta resuelve y "off shoring" (basada en el servicio) en que la Universidad contrata una unidad tercerizada y los desarrollos los realizan proveedores asistidos por la universidad para hacer una transferencia.

Por otra parte, a quienes creen en los beneficios de la aplicación de la parte gestión de calidad, una vez que se implementan estos modelos se asigna importancia a la evaluación de cómo van impactando los mismos en la sociedad bajo la premisa que la evaluación invierte la ecuación de la transferencia investigación $\square$ extensión en extensión $\square$ investigación; creación de instituciones que promuevan la Extensión. En la FI se creó el Instituto de tecnología industrial que englobó al INTEMI (Instituto Minero) de la Facultad de Ingeniería, el cual depende del Decano y a través del cual se lleva a cabo el gerenciamiento de la extensión con implicancias en todas las extensiones; desarrollar tecnología en tres etapas escala, piloto y final articulando los desarrollos con las empresas como financiadores y luego ir a un acuerdo de coparticipación de patentes; formalizar y sistematizar los antecedentes por actividades de extensión para dotarlas de capital simbólico para su acreditación y entre otras cosas compensar la falta de estímulo económico que es observada como una falencia de las mismas, tema que se encuentra a su vez en cierta tensión dialéctica con que, por otro lado, la transferencia acredita y da estatus en la universidad porque "no cualquiera puede hacer transferencia con empresas"; y la propuesta de realizar flujogramas para los proyectos en el que se visualicen los vínculos del proceso extensionista. 


\subsection{Precategoría: actividades de alto/bajo impacto evocadas en materia de extensión}

Se encontraron 13 fragmentos de entrevistas vinculados a esta precategoría que se espera que resulten orientadoras cuando más adelante se trabaje con la contraparte, es decir la visión que tienen las organizaciones impactadas por la extensión de la UNJU y cómo se teje la trama de la demanda considerando las dos partes que intervienen en el proceso. En la FCA se destacan como actividades de bajo impacto las expectativas con las intervenciones escolares que se diluyen por la imposibilidad de mecanismos y recursos para evaluar su efecto multiplicador en la comunidad porque la tarea educativa es a largo plazo, hoy se habla con niños de quienes luego se desconoce su trayectoria. Se habla de alto impacto en la parte de gastronomía y turismo con organizaciones gastronómicas y chef que luego dieron lugar a presentaciones en congresos y simposios, por ejemplo sobre maíz andino. Por otra parte, el recordatorio de un relevamiento hecho en los lotes de una finca (el Pongo) con alumnos de la FCA mostraría un modelo de trabajo allí donde se necesitaba capacidad técnica y dedicación de un organismo que no fuera el Gobierno, ya que este genera desconfianza respecto a que los pudieran evaluar patrimonialmente.

En el caso de la FI se evoca el caso de una intervención de cuatro años en una empresa cementera que muestra cómo a partir del desgaste de un área de una empresa se puede hacer un emprendimiento (súper laboratorio) y sustentar metodológicamente lo que se hace desarrollando estructura técnica en la gente (a pocos días de acreditar un laboratorio a nivel Nación, que en Jujuy no hay) haciendo participar a los estudiantes en los trabajos. También, en grupos que trabajan aromáticas y alimentos $\square$ inversión, un entrevistado observa las dificultades para vincular la potencialidad que hay con los requerimientos de las empresas del medio donde el concepto del investigador de la FI es que su producto es para redactar papeles sin que llegue en profundidad a la sociedad; en la misma línea, está el caso de investigaciones sobre la arcilla de Jujuy para transferir información a los artesanos como apoyatura. Otros emprendimientos destacados son la creación de programas como el de apoyo al microemprendedor, convenio donde se destina financiamiento para un equipo de seis profesionales, "senior" y "junior", con la perspectiva de llegar con alumnos avanzados para configurar una experiencia que los convierta en potenciales microemprendedores y el Voluntariado Universitario (Programa Nacional de la Secretaría de Políticas Universitarias de la Nación, 2006).

\subsection{Códigos y categorías emergentes}

Se organizaron los códigos clasificándolos en dos categorías que remiten respectivamente a 2 dimensiones 8 de la extensión: normativa y económica:

\subsubsection{La dimensión normativa de la extensión}

En los dichos de los interpelados aparece una alusión respecto a que en cierto modo la extensión posee más certidumbre en regulaciones externas que internas en las UU.NN., como la Ley Nacional de Vinculación de la República Argentina, las Normas ISO internacionales para aplicar a la gestión de calidad de acciones y contenidos de las mismas con referencias a ausencias o insuficiencias de un marco formal para desarrollar políticas de Extensión y desconocimiento de cuáles son los marcos regulatorios de las Facultades o la Universidad aplicables a la extensión. También aquí se hace referencia 
a que las cuestiones de mediano y largo plazo conviene desarrollarlas por convenios a nivel rectorado observando los alcances y limitaciones de trabajos que signifiquen un desarrollo constructivista para la empresa y una innovación tecnológica considerando los potenciales riesgos ${ }^{9}$.

\subsubsection{La dimensión económica de la extensión}

Un elemento que problematiza fuertemente es el del arancelamiento en la extensión (aparece siempre con redundancia la temática de la capacitación): si por la tarea extensionista debe cobrarse o no, los mecanismos que deberían utilizarse para el manejo de fondos y las posibles soluciones al respecto como facturar a través de una cooperadora o una Fundación para generar una unidad de negocios que permita salir de la burocracia de los cánones de comprar por la universidad.

Por otra parte, también se identifican como obstáculo para el arancelamiento a los docentes que no quieren inscribirse en la Administración Federal de Ingresos Públicos para facturar servicios a cuenta de la Universidad, (que funciona cómo agente recaudador ante el órgano impositivo) visualizándose como un segundo obstáculo a los grupos de investigación que funcionan de manera cerrada para intentar constituir unidades de negocios, los que, por otra parte son potenciales destinatarios de los fondos captados.

\section{Conclusiones}

Hacia el final del análisis preliminar, en una codificación axial a partir de buscar reagrupar códigos y categorías surgidos de la codificación abierta, y a partir de los códigos emergentes reunidos en lo que se dio en denominar como las dimensiones Normativa (definida por su correspondencia con la base legal que regula la extensión) y la Económica (los aspectos presupuestarios y financieros que sostienen a la extensión), se encontró que las precategorías y subcategorías derivadas de las anteriores también se podían congregar $\square$ siguiendo una lógica similar $\square$ en otras dos dimensiones que se denominaron como dimensión académica (que se refiere a los planos epistemológicos, metodológicos, teóricos y/o teórico-prácticos de la extensión) y una dimensión política de la extensión (vinculada a los aspectos que hacen al gobierno y organización de la extensión). Ello permitió relacionar y subsumir las categorías a priori dentro de estas categorías a posteriori quedando entonces: 1) en la dimensión académica de la extensión: concepto de extensión que orienta la práctica, características asignadas a los demandantes, formación sobre demanda, estrategias extensionistas; 2) en la dimensión política de la extensión: ámbitos, organizaciones y agentes con los que se realiza la vinculación, las formas y vías como se tratan pedidos y acciones de extensión. Más difícil resultó encuadrar la precategoría actividades de alto/bajo impacto evocadas en materia de extensión puesto que aquí los entrevistados caracterizan a las mismas por la propiedad del efecto formador que tienen las mismas en los agentes de la extensión, lo que la ubicarían en la dimensión académica o por el alcance transformador sobre los destinatarios que la ubicaría en la dimensión política.

Ahora bien, ¿cuáles son los hallazgos en estas dimensiones y categorías con sus propiedades? ¿Qué elementos discursivos de los informantes clave pueden constituir un aporte a la construcción de una teoría de la práctica extensionista que luego pueda interpretarse y examinarse críticamente para ver qué y cuánto de ello puede volver a esta práctica para convertirla en una praxis informándola teóricamente?

En la dimensión académica y respecto al concepto de Extensión en las ingenierías aparece una concepción marcadamente vinculacionista, lo que no constituye una primicia (López, 2005, p. 16), donde el lucro 
juega un papel variable mayor en la FI que en la FCA más emparentado a generar una unidad de negocios que proporcione dividendos en la primera y en ambos casos relacionado con la prestación de servicios a terceros (de tipo repetitivo) o actualizaciones y transferencias provenientes de la investigación. La posible novedad aquí es, en todo caso, ubicar una tercera conceptualización de extensión, que sigue siendo vinculacionista, concebida como desarrollos adaptados a la organización que se realizan con el "otro" que puede ir más allá del trabajo de las modalidades de transferencias de la investigación y la mera prestación de servicios repetitivos. Identificar estos elementos para incorporarlos a una teoría extensionista puede ser orientador para quienes asuman responsabilidades respecto al entramado de factores y antagonismos propios de las prácticas y representaciones en el área que incluyen los procesos de demanda.

La mayor redundancia de la actividad extensionista que aparece en todos los entrevistados está referida a la capacitación. Para explicar la complejidad que tiene el escalonamiento de la capacitación en la extensión se crearon conceptos, que pueden tener alcance teórico, cómo doble transferencia, y transferencia primaria $\mathrm{y}$ transferencia secundaria ${ }^{10}$. Considerando la categoría características asignadas a los demandantes, subsumida en la misma dimensión académica, se ve aquí diferencias en el caso de la FI y la FCA. En la FI los agentes demandantes considerados son principalmente un Estado burocrático y la gran empresa, caracterizada como más dinámica que el anterior, ambos posibles fuentes de recursos para los negocios, emergentes de un contexto social con una mentalidad prebendaria que exige tomar recaudos.

En el caso de la FCA, si bien se articula y no se descarta la gran empresa y el Estado, el foco está puesto en un destinatario de servicios como el agente rural más de base constituido por el pequeño productor campesino o aborigen en que la FCA ha tomado parcialmente un rol (más allá de lo productivo) como promotor cultural de la región en la que impacta. Se descubre además un agente advenedizo destinatario de la extensión que es el turismo sobre todo en lo relacionado al plano agroalimentario. Si bien se parte de un objeto de investigación que se pretende esclarecer, la relación demanda-extensión, el cual tenía en principio como parte a la comunidad interna y externa sujeto de la demanda extensionista y como contraparte a la universidad y los docentes/investigadores universitarios, ya en este avance, de lo dicho, surgen como categorías que dan complejidad a este concepto respectivamente: el rol del Estado, el rol de la empresa, el rol de las organizaciones desde la sociedad civil y los particulares en la definición y/o construcción de la demanda extensionista y otros sujetos que son los funcionarios y exfuncionarios de extensión y los docentes/ extensionistas que no hacen investigación.

Todo ello remite a otra categoría: cuál es el rol de las unidades académicas en materia extensionista en el plano de la política general universitaria en la etapa de la demanda y más allá de ella y qué antagonismos y acuerdos pueden favorecer y obturar el accionar. Es en este marco y a partir de esta caracterización que por lo menos algunos actores de la FCA muestran un modelo para jugar un posible papel en el apuntalamiento de las particularidades culturales de las comunidades locales en materia agropecuaria. Relacionado con lo anterior y continuando en la dimensión académica de la extensión en el rubro Estrategias extensionistas, se puede vislumbrar un panorama de procedimientos efectivizados o proyectados desde las ingenierías que con distintos grados de originalidad se constituyen en ideas y potenciales aportes al campo metodológico de la Extensión para otras carreras de ingenierías e incluso otros campos disciplinares. En la categoría Formación sobre demanda, 
una pista significativa respecto a donde se puede recabar formación/información queda referida en el campo de la Psicología laboral.

Continuando hacia la dimensión política de la extensión y contemplando la tabla del punto 3.2 se puede obtener una idea parcial de algunos ámbitos y organizaciones con los que se vinculan las ingenierías de la UNJU, lo que permite conocer lo que pasa y pensar y repensar los ámbitos de la vinculación.

Respecto a formas y vías como se tratan pedidos y acciones de extensión, que se han ubicado en la dimensión política de la extensión, los entrevistados referencian el seguimiento de distintas vías (lo que en el análisis de la categoría 1 se definía como extensión orgánica) que muestra la posibilidad de convivencia de una organización con vías formales (extensión orgánica) e informales (extensión inorgánica), en las que tiene protagonismo la actitud que toman las autoridades de los equipos de conducción en los más altos niveles respecto a cómo se articulan con las áreas de extensión, el rol que les es asignado a estas y las estructuras con las que se organizan dichas áreas como factores determinantes en el tipo de vínculos que se establecen con la comunidad.

En síntesis y volviendo al problema y objetivos iniciales de la investigación respecto al tratamiento que tiene la demanda en materia de Extensión en distintos ámbitos de la universidad pública y la posibilidad de vincular estilos de abordaje de la demanda con procesos y resultados, se encuentra que al teorizar $\square$ inicial y provisoriamente $\square$ la práctica desde un recorte de las ingenierías, la extensión universitaria y la demanda en dicha extensión como uno de sus aspectos constitutivos, se puede interpretar desde dimensiones relevantes: académica, política, normativa y económica, con sus respectivas características (propiedades analizadas), de las cuales las primeras dos se construyen a partir de una serie de precategorías que se consideraron inicialmente como puntos de partida y que se confirman como relevantes y otras dos que emergen del discurso de los informantes, todas, de una otra manera, potencialmente claves para la dilucidación de la Extensión Universitaria como problema.

\section{Notas}

1 Códigos C-110/09 Se.C.T.E.R-U.N.Ju. y 08/C185/09 Programa de Incentivos.

2 "Universidad Nacional de Jujuy 30 años de Historia”, publicación de la Universidad. Jujuy, Ediunju, 2004

3 Al 31 de marzo de 2010. Información suministrada por la Coordinación Académica de la Secretaría de Asuntos Académicos de la UNJU.

4 Temática desarrollada en un artículo actualmente en trámite de publicación.

5 La muestra intencional comenzó con (ex) funcionarios de extensión que a su vez derivaron a docentes/investigadores considerados representativos de la extensión que se hace en cada facultad como puntapié inicial del muestreo teórico que requiere la perspectiva de la teoría fundamentada en los datos. También, en la aplicación del método comparativo constante que requiere dicho enfoque en este avance parcial, en un primer momento se establecieron similitudes entre la extensión que realizan la FCA y la FI para luego determinar diferencias en los estilos que tienen estas unidades en el tratamiento de la demanda en Extensión, lo que se observa hacia el final del trabajo.

6 Aunque fue remitida una primera versión con los fragmentos de entrevistas antecediendo a los códigos y categorías que se iban extrayendo como material probatorio, si bien este material es fundamental para la evaluación dentro de una perspectiva de investigación cualitativa (por ejemplo en el informe final del trabajo) el mismo se suprimió en atención a la observación que fragmentaba mucho la lectura en un espacio limitado como lo es el de una publicación. Sólo se han dejado incorporadas las citas textuales en el análisis de la primera categoría a modo de ejemplo de la mecánica de trabajo.

7 No me refiero aquí a "cosas fundadas" sino a las asociaciones civiles sin fines de lucro que funcionan bajo esta figura legal.

8 Es necesario aclarar que las "dimensiones" aquí no se refieren a las dimensiones de las propiedades de las categorías sino que 
el término se ha integrado al nombre de las categorías (la dimensión normativa, la dimensión, económica, etc.).

Se cita el ejemplo de río IV, en la provincia de Córdoba, accidente con víctimas fatales ocurrido en el transcurso de una investigación que llevaba adelante la universidad con una empresa.

10 Referida a la capacitación que hacen los extensionistas a los agentes destinatarios de la extensión que se denomina transferencia primaria y la socialización y los efectos multiplicadores de la misma que hacen dichos agentes en nuevas instancias donde por ejemplo se realizan nuevas capacitaciones a terceros, trasferencias en algún tipo de congreso, etc. a lo que se denomina transferencia secundaria.

\section{Referencias bibliográficas}

Bleger, J. (1999). Psicohigiene y Psicología Institucional $\quad\left(7^{\mathrm{a}}\right.$ reimpresión). Buenos Aires: Paidós.

Butelman, I. (1987). Psicopedagogía Institucional. Buenos Aires: Paidós.

Fernández, G. (2003). Estrategias de Intervención Institucional. En de Mezzano, Alicia N. C. Psicólogos Institucionales Trabajando. La psicología institucional en docencia, investigación y extensión universitaria (225-256). Buenos Aires: Eudeba.

López, M. (2010). University Extension. Problem Identification and Guidance for Managing the Area. Revista de Universidad y Sociedad del Conocimiento (RUSC) 7 (2) UOC. Recuperado de http://rusc.uoc.edu/ ojs/index.php/rusc/article/view/v7n2lopez/v7n2-lopez-eng

López, M. (2005). Extensión en el Nivel Superior. Experiencia desarrollada en la FHYCS-UNJU. Jujuy, Argentina: EdiUnju Jujuy.

Lourau, R. (1991). El Análisis Institucional (2a Reimpresión). Buenos Aires: Amorrortu Editores.
Mendizábal, N. (2007). Los componentes del diseño flexible en la investigación cualitativa. En Vasilachis de Gialdano, Irene Estrategias de Investigación Cualitativa (65-106). Buenos Aires: Gedisa.

Pampliega de Quiroga, A. (1994). Enfoques $y$ perspectivas en psicología social. Buenos Aires: Ediciones Cinco.

Pichon, E. (1985). El Proceso Grupal. Buenos Aires: Ed. Nueva Visión.

Programa Nacional de Voluntariado Universitario de la Secretaría de Políticas Universitarias de la Nación (2006) Recuperado de http:// www.me.gov.ar/spu/guia_tematica/ VOLUNTARIADO/voluntariado.html

Quiroga, L. (2001) Apuntes para la historiografía de la extensión universitaria (interacción social) VI Congreso Iberoamericano de Extensión Universitaria, EMBU, San Pablo, Brasil. Recuperado de http://www. umss.edu.bo/Academia/Direcciones/ Disu/aphistoriog.htm

Rodríguez, G., Gil, J. y García, E. (1996). Metodología de la investigación cualitativa. Málaga, España: Ed. Aljibe.

Sirvent, M. (1997). Breve diccionario Sirvent. Conceptos iniciales básicos sobre investigación en Ciencias Sociales. Buenos Aires: Editado por la Maestría en Didáctica de la Facultad de Filosofía y Letras de la UBA.

Sirvent, M. (1996). Educación de jóvenes y adultos en contexto de ajuste. En Revista del IICE. 5, (9) 65-77. Buenos Aires: Miño y Dávila Editores.

Soneira, A. (2007). La teoría fundamentada en los datos (Grounded Theory) de Glaser y Strauss. En Vasilachis de Gialdano, Irene Estrategias de Investigación Cualitativa (153-174). Buenos Aires: Gedisa. 